\title{
Drug delivery system for an anticancer agent, chlorogenate-Zn/Al-layered double hydroxide nanohybrid synthesised using direct co-precipitation and ion exchange methods
}

\begin{abstract}
A nano-structured drug-inorganic clay hybrid involving an active anticancer compound, which is chlorogenic acid (CA) intercalated into $\mathrm{Zn}$ /Al-layered double hydroxide, has been assembled via ion-exchange and co-precipitation methods to form a nanohybrid CZAE (a chlorogenic acid-Zn/Al nanohybrid synthesised using an ion-exchange method) and CZAC (a chlorogenic acid-Zn/Al nanohybrid synthesised using a direct method), respectively. The Xray diffraction $(\mathrm{XRD})$ results confirmed that the $\mathrm{CA}-\mathrm{LDH}$ had a hybrid structure in which the anionic chlorogenate is arranged between the interlayers as a horizontal monolayer at 90 and $20^{\circ}$ angles from the $\mathrm{x}$ axis for CZAE and CZAC, respectively. Both nanohybrids have the properties of mesoporous materials. The high loading percentage of chlorogenic acid (approximately $43.2 \%$ for CZAE and $45.3 \%$ for CZAC) with basal spacings of 11.7 and 12.6 $\AA$ for CZAE and CZAC, respectively, corroborates the successful intercalation of chlorogenic acid into the interlayer gallery of layered double hydroxides. Free chlorogenic acid and the synthesised nanocomposites (CZAE, CZAC) were assessed for their cytotoxicity against various cancer cells. The Fourier transform infrared data supported the formation of both nanohybrids, and a thermal analysis showed that the nanohybrids are more thermally stable than their counterparts. The chlorogenate shows a sustained release, and the release rate of chlorogenate from CZAE and CZAC nanohybrids at $\mathrm{pH} 7.4$ is remarkably lower than that at $\mathrm{pH} 4.8$ due to their different release mechanisms. The release rate of chlorogenate from both nanohybrids can be described as pseudo-second order. The present investigation revealed the potential of the nanohybrids to enhance the in vitro anti-tumour effect of chlorogenic acid in liver and lung cancer cells in vitro.
\end{abstract}

Keyword: Zn/Al-layered double hydroxides; Co-precipitation method; Ion-exchange method; Chlorogenic acid; Cancer cells 